\title{
Topology-Aware Distributed Smart Grid Control using a Clustering-Based Utility Maximization Approach
}

\author{
Marius Stübs \\ University of Hamburg \\ Hamburg, Germany \\ stuebs@informatik.uni-hamburg.de
}

\author{
Hanko Ipach \\ Hamburg University of Technology \\ Hamburg, Germany \\ hanko.ipach@tuhh.de
}

\author{
Christian Becker \\ Hamburg University of Technology \\ Hamburg, Germany \\ c.becker@tuhh.de
}

\begin{abstract}
Efficiently balancing supply and demand in the electrical distribution grid is an important Smart Grid service. The development of applicable agent-based concepts for match-making energy generators with consumers is an active research field. Implementing Smart Grids as a system of Micro Grid systems, we propose an efficient decentralized optimization scheme, which avoids central components in order to increase dependability, robustness against communication failures, and denial-of-service attacks. The concept utilizes traditional peer-to-peer functionality in combination with locality-aware routing: Two explicit clusterings are evaluated and compared to the fully-meshed communication approach. We investigate the impact of node clustering on the optimality and locality of the distributed Smart Grid control algorithm and show that our approach significantly reduces the electrical losses in the examined power distribution grid compared to the centralized approach.
\end{abstract}

\section{CCS CONCEPTS}

- Computer systems organization $\rightarrow$ Peer-to-peer architectures; Fault-tolerant network topologies; • Computing methodologies $\rightarrow$ Agent / discrete models;

\section{KEYWORDS}

Smart Grids, System of Systems, Cyber-Physical System, Distributed Control Algorithm, Topological Clustering, Utility Maximization, Resilience, Controller-Hardware-in-the-Loop, Iterative Optimization, Electrical Power Balancing

\section{INTRODUCTION}

The electricity distribution system is in a transition. Due to the ubiquitous introduction of renewable energy sources, power generation is organized more and more in decentralized ways, often using volatile energy forms that are hard to predict. A large number of renewable energy generation units is installed in the electrical distribution grids on the medium voltage (MV) or low voltage (LV) levels. The increasing share of controllable loads such as battery storages, heat pumps or other inverter-based power systems additionally amplifies the need for fast and precise secondary control schemes in distribution grids as well as their applicability. This

Permission to make digital or hard copies of part or all of this work for personal or classroom use is granted without fee provided that copies are not made or distributed for profit or commercial advantage and that copies bear this notice and the full citation on the first page. Copyrights for third-party components of this work must be honored For all other uses, contact the owner/author(s).

SAC '20, March 30-April 3, 2020, Brno, Czech Republic

(C) 2020 Copyright held by the owner/author(s).

ACM ISBN 978-1-4503-6866-7/20/03.

https://doi.org/10.1145/3341105.3373959 makes the control of electric power generation and consumption in distribution grids an important research topic.

Micro Grids define independent sections of the distribution grid, consisting of power generators and power loads, as well as battery storages and power conversion systems like power-to-gas appliances [20]. For our model, we assume a fully meshed communication network.A utility factor is associated to each node, indicating their contribution to the overall systems welfare per consumed or produced power unit. The more generic concept of focusing on utility or social welfare instead of costs has recently gained attention in electric power systems [16].

As a realistic scenario for the simulation, we utilize the IEEE European Low Voltage Test Feeder as shown in Figure 1, where each agent represents one Distributed Energy Resource (DER). The red circles mark the electric lines where critically high power flow peaks are expected.

\subsection{System of Systems Perspective on Smart Grids}

The term "System of Systems" (SoS) describes a constellation of independent systems that, despite their heterogeneity and independent development, collaborate and yield emergent properties [10]. The use of Smart Grid agents, representing either producers or consumers, is an innovative take towards dynamic and dependable software-intensive system of Smart Grid systems [11]. Balancing

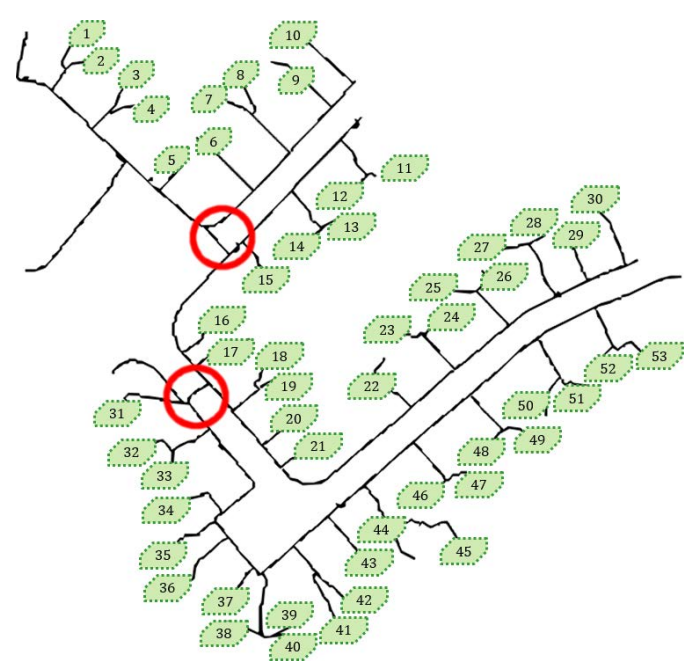

Figure 1: IEEE European Low-Voltage Test Feeder Grid. Red circles mark critical connections. 
production and consumption is usually realized either by a central control algorithm or by a transparent market, where offers are published, then bids are placed and finally the winner gets to produce more or to consume more. Global market schemes do not regard locality and cause high transmission losses and congestion on the electric power lines. In both cases, the calculation of the optimal power flow in the Micro Grid is usually not feasible in real time [21].

\subsection{Contribution}

The contributions in this paper are:

a) a novel approach on Smart Grid power balancing as a SoS,

b) the introduction of a utility factor concept into the domain of distributed Smart Grid control,

c) an elaboration on locality-centric research for global optimization problems using a clustering-based appraoch, and

d) an algorithm for reducing power line losses without explicitly considering the electricity grid parameters (e.g. line impedances), except for topology information.

\subsection{Structure of the Paper}

Within this paper, we propose a distributed algorithm for resilient Smart Grid control. It is structured as follows: Section 2 details the fundamental requirements of the proposed algorithm and introduces the important concepts of utility-driven optimization and flexibility modeling. Section 3 compares our approach to related work. In Section 4, a distributed Smart Grid control scheme for resilient match-making, consisting of four consecutive steps, is elaborated. Simulation results are presented in Section 5, elaborating on the effects of different communication overlay topologies. In Section 5.4 and 5.5, two different clustering schemes are evaluated for their impact on the investigated Micro Grid's power flow. In Section 6 we conclude with an evaluation of the algorithm with regards to the postulated requirements and an outlook on our next steps.

\section{BASIC CONCEPTS AND REQUIREMENTS}

This section describes the requirements of distributed Smart Grid control schemes as well as fundamental concepts underlying the developed algorithm.

\subsection{Electric Power Grid Constraints}

The expansion of generation and consumption capacity within the distribution grids implies that overload situations are becoming more likely, i.e. currents exceeding the thermal limit of power lines or transformers or node voltage deviations exceeding the admissible range. Due to high costs related to e.g. the installation of new power cables, the prevention of inadmissible electric power system states by means of control algorithms is promising compared to empowering the grids. In the following, we refer to constraints arising from current or voltage limitations as the power grid constraints.

Within electric power grids, the challenge of optimizing overall costs, social welfare or grid losses by adjusting the power output and/or consumption of flexible devices while taking power grid constraints into account is commonly referred to as optimal power flow $(O P F)$ problem. The major hurdle when tackling an OPF problem is that the power flow equations relating node voltages and line currents to power injections are non-linear and non-convex, therefore complicating the optimization problem. Recent approaches make use of convex relaxations [8] or linearizations [1] to facilitate the optimization problem. While these simplifications decrease computation time, a complete model of the power grid is still required by OPF algorithms.

In contrast to the OPF algorithms, the distributed Smart Grid control algorithm proposed in this paper is agnostic of the electrical parameters of the power grid connecting the various agents. It therefore neglects power grid losses and constraints. Though not explicitly considering grid constraints, we propose a distributed algorithm that is inherently grid-serving, as it tries to match power supply and demand on a local scale. It thus avoids the lossy transmission of electrical power over long distances.

\subsection{Agent-based Smart Grid Control}

From the perspective of SoS, we identify the following properties for our algorithm to implement:

Locality-Aware: Each node has its local view of the system state. The distributed algorithm should come up with a near-optimal solution nonetheless.

Tie-Breaking: Each two nodes in the network that compete for resources should have a definite rule-set to decide which node to prefer or how to split the resource.

Scalable: When confronted with a Micro Grid comprising uncommonly many DERs, an optimal solution should be found in a reasonable time.

Iterative: The optimization scheme should consider the current state of the system and apply changes iteratively to find the optimal solution.

Measurable: The optimality of any solution should be quantifiable and any two solutions should be comparable in terms of the system utility.

\subsection{Clustering-based System of Systems}

Solving the balance requirements for each cluster as an independent sub-system increases the resilience and fosters the system-ofdependable-systems approach [3]. The clusters are designed to have an overlap where one or more nodes in each cluster are additionally members of another cluster. These nodes mediate the imbalance in power generation/consumption between their parent clusters. Figure 2 depicts the concept of clustering nodes to define their explicit neighborhood, where nodes that belong to overlapping clusters

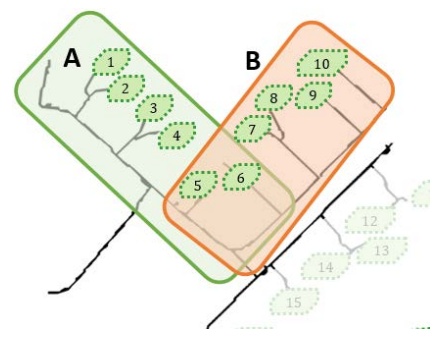

Figure 2: Two overlapping clusters 
can offer their capabilities in both of them and thus implicitly optimize/negotiate between them. Clustering has several advantages: By splitting the original problem into the smaller systems, local solutions are given priority. This also increases the resilience of the overlying system against denial-of-service-attacks, because any cluster can reach a balance in itself when the connection to more distant nodes fails. Even despite failure of single nodes, all available nodes contribute to their means. Message complexity of the algorithm decreases since a) the broadcast announcement of power consumption or generation offers is restricted to the parent cluster and b) since the single clusters are balanced before escalating, only the remaining imbalance has to be communicated to other clusters.

\subsection{Utility-Driven Optimization}

In the context of Smart Grids, we define the utility factor to quantify the value of electric power consumption (resp. production) for a certain consumer (resp. producer). It is a generic concept which might take individual as well as social aspects into account. In the remainder of this paper we assume that the utility factor of a windmill generating electricity is generally high, since there is no cost associated with its operation, whereas CHP plants are usually more costly which results in a lower utility. At the same time, the utility factor of a hospital is higher than that of a charging station for electrical cars due to its higher importance for the society. Furthermore, the utility factor in our model is not only connected to the type of producer or consumer, but also to the urgency of the offer. In case of a large power abundance at a node, the utility factor of selling the unneeded power is higher than when the divergence is small. Therefore, similar appliances can have different utility factors - e.g. battery storages due to their different charging states and operator preferences.

At any time, the absolute utility $u_{a}(n)$ of a node $n \in N$ is calculated by multiplying the utility factor $\mathrm{uf}(n)$, which is assigned to each DER, with its power output $p(n)$ :

$$
u_{a}(n)=\operatorname{uf}(n) \cdot p(n) .
$$

The sum of all node utilities then adds up to the overall utility $U$ of that specific optimization solution:

$$
U=\sum_{n \in N} u_{a}(n) .
$$

The amount of power by which a consumer or producer is able to increase or decrease its power output or consumption is also referred to as flexibility. The flexibility of electrical cars is usually higher than that of a hospital, since electrical cars are a typical example of demand response applications [13] and can generally shift their charging period as needed by the distribution grid operator [17].

\section{RELATED WORK}

Recent research on distributed control in electric grids has brought up a number of approaches [15, 16, 23]. A variety of distributed algorithms for optimization in electric power system has been developed [12, 22]. Nonetheless, a research gap is noticeable in the field of agent-based locality-aware optimization approaches that take clustering into account as a possible topological decision. Our research contributes to this area.

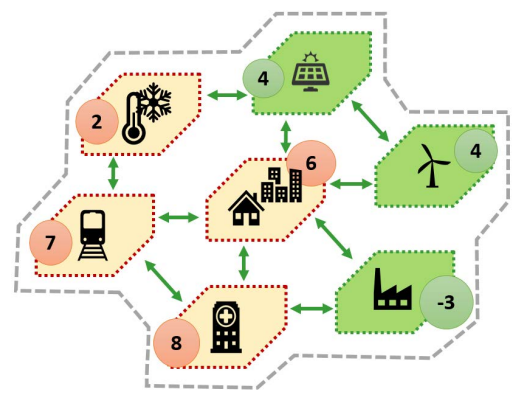

Figure 3: Example Micro Grid with seven agents. The numbers indicate the node specific utility factor.

The investigation of Smart Grids as a SoS is usually presented from perspective where functionality is separated in various layers $[9,14]$. Our approach extends this view by introducing a clustering approach, where each cluster in the optimization scheme itself can be seen as a subsystem.

Utility-based optimization is can be categorized into the field of economic dispatch [7] and has also been studied in communication networks as a concept to allocate limited resources fairly [2]. Here, we apply it to electric power grids, transferring the idea of fairness to scenarios with limited power consumption and generation capacity.

A fully decentralized social welfare optimization algorithm based on a utility concept is proposed in [16], considering demand and generation side simultaneously and neglecting grid constraints. In contrast to our approach, a convex optimization problem is formulated to maximize the overall utility which requires local price regulators in its distributed solution. In [4], a social welfare optimizing two-level auction mechanism for power allocation in distribution grids is presented. Power grid constraints are incorporated in a simplified manner, neglecting grid losses and requiring a radial grid topology. This limitation is not required for our algorithm, which is applicable independent from the grid structure.

The use of clustering on Micro Grids using software-defined networking (SDN) for resilient distributed power sharing has already been shown [18]; our approach presents a complementary view with the perspective from electrical grid properties and the aim for avoiding any central components on the distributed control layer.

\section{MATCH-MAKING ALGORITHM}

We now describe the distributed Smart Grid control scheme, which acknowledges the requirements as well as the electrical power grid constraints as defined in Section 2.

Figure 3 shows an example scheme with seven nodes. Four consuming nodes, or loads, identifiable by their brighter yellow background color, represent a) public transportation, b) private households and offices, c) a hospital and d) an industrial cooling system. The associated numbers represent the utility factor of the respective appliance. In this example we chose a scale between -9 and +10 , where -9 means undesirably costly and 10 stands for critical appliances. Any two appliances would mutually exchange power, when the sum of their utility factors is above zero, which means that the overall system's utility would increase in this scenario. 
The three producing nodes represent a PV-panel, a wind turbine and a CHP plant. The negative 3 at the CHP plant signifies that it would be activated if loads with a larger utility than 3 require it to. Note that the industrial cooling system currently has a utility of only 2. In the current configuration, the CHP plant is not activated to supply the demand of the cooling system, because their combined utility is below zero. This might change as soon as the cooling system reaches an internal threshold where further in-action would lead to a critical state. Then the priority of cooling would increase, leading to a higher utility factor which in turn would activate the CHP.

We defined the term flexibility as the possible adjustment of generation or consumption that is available at a specific node. It is expressed in terms of electric power. For example, a combined heat and power plant (CHP plant) has a flexibility with a high grade of granularity, while photo-voltaic panels (PV-panels) without sophisticated control devices might only be turned on or off.

The conceptional description in Figure 4 is based on decentralized events that occur at independent positions in the grid. The trigger event can be an external change in the physical environment, e.g. an increasing wind that allows the DER to announce a higher flexibility to other nodes. It is important to note that after each agreement, the potential flexibility of both involved nodes has changed; therefore, a trigger event is sent, initiating a new round of the algorithm.

\subsection{Pairwise Agreement}

Let $\mathcal{N}$ denote the set of nodes, with $n=|\mathcal{N}|$. The nodes are grouped into $m \in \mathbb{N}$ (not necessarily disjoint) clusters $C_{1}, \ldots, C_{m}$ such that $\mathcal{N}=C_{1} \cup \cdots \cup C_{m}$.

The match-making process comprises three steps: First, a node $n_{a} \in C_{i}$ (the announcer) publishes to the current cluster $C_{i}$ the information that its flexibility changed. If the announcement is relevant to some neighboring nodes $n_{r} \in C_{i}$ in the same cluster $C_{i}$, each of them replies with an according offer $o$. We call these nodes the replying nodes. Then the announcer node $n_{a}$ decides which of the offers to select and answers with a settlement message $s$, which validates the agreement between both parties.



Figure 4: Procedure for reaching an agreement
The inner state of each node consists of two flexibilities: The positive flexibility $\mathrm{f}^{+}$includes the value how much power $\mathrm{p}^{+}$it is able to sell, in other words its capacity to increase power injection, and the associated utility factor $\mathrm{uf}^{+}$. The negative flexibility $\mathrm{f}^{-}$ contains the capacity $\mathrm{p}^{-}$to either reduce injection or increase power consumption and the respective utility factor $\mathrm{uf}^{-}$:

$$
\begin{aligned}
& \mathrm{f}^{+}=\left\langle\mathrm{p}^{+}, \mathrm{uf}^{+}\right\rangle, \\
& \mathrm{f}^{-}=\left\langle\mathrm{p}^{-}, \mathrm{uf}^{-}\right\rangle .
\end{aligned}
$$

4.1.1 Flexibility Announcement. An agent whose Distributed Energy Resource (DER) changes in its possible output announces the resulting flexibility. The announcement $a$ therefore contains two flexibilities $\mathrm{f}_{a}^{+}$and $\mathrm{f}_{a}^{-}$:

$$
\mathrm{a}=\left\langle\mathrm{f}_{a}^{+}, \mathrm{f}_{a}^{-}\right\rangle
$$

This information is distributed to the other nodes in the Micro Grid. Because of the clustered scheme, only nodes in the same cluster $C_{i}$ are informed about the change and the message overhead is reduced compared to a full broadcast.

4.1.2 Offering a Match. Each node $n_{r}$ in the cluster $C_{i}$ calculates the resulting utility factor for a deal by combining its own flexibilities and utility factors with the received announcements: $\mathrm{uf}_{o}^{+}$ describes the expected utility of an offer $o^{-}=\left\langle\mathrm{p}_{r}^{-}, \mathrm{uf}_{r}^{-}\right\rangle$that could be sent to the announcing node $n_{a}$, offering to consume the advertised power injection. $\mathrm{uf}_{o}^{-}$on the other hand describes the expected utility factor of an offer $o^{+}=\left\langle\mathrm{p}_{r}^{+}, \mathrm{uf}_{r}^{+}\right\rangle$to lower its own consumption or increase its own power injection to the grid.

$$
\begin{aligned}
& \mathrm{uf}_{o}^{+}=\mathrm{uf}_{a}^{+}+\mathrm{uf}_{r}^{-} \\
& \mathrm{uf}_{o}^{-}=\mathrm{uf}_{a}^{-}+\mathrm{uf}_{r}^{+}
\end{aligned}
$$

The availability of additional electric power in the Micro Grid can be out-balanced in two ways: Either the consumer with the highest utility factor can offer to increase its consumption or any generator with a lower utility factor (corresponding to a higher energy cost price) can offer to reduce its generation.

In addition to the utility factor, the amount of power exchange is calculated:

$$
\begin{aligned}
& \mathrm{p}_{o}^{+}=\min \left(\mathrm{p}_{a}^{+}, \mathrm{p}_{r}^{-}\right) \\
& \mathrm{p}_{o}^{-}=\min \left(\mathrm{p}_{a}^{-}, \mathrm{p}_{r}^{+}\right)
\end{aligned}
$$

All consumers and generators select an offer that satisfies the requirements of being

not empty: $\mathrm{p}_{o}>0$, and having

positive utility: $\mathrm{uf}_{o}>0$.

If more than one offer is not empty and has a positive utility, the offer with the highest utility is selected. Node $n_{r}$ then communicates the selected offer $o$

$$
\mathrm{o}=\left\langle\mathrm{p}_{o}, \mathrm{uf}_{o}\right\rangle
$$

to the agent $n_{a}$ who had initiated the trade.

Note that this step has a blocking behavior: Each flexibility offer has a short expiry period until the offering node is committed to its offer. 
4.1.3 Settlement. In the third step, the announcer $n_{a}$, has to accept one of the offers $o_{r}=o_{r}^{+} \vee o_{r}^{-}$from one of the responders $n_{r} \in R$, to increase its power generation.

First, the possible settlements $s_{r}=\left\langle\mathrm{p}_{s, r}, \mathrm{uf}_{s, r}\right\rangle$ are generated.

$$
\begin{aligned}
& \mathrm{p}_{s, r}^{+}=\min \left(\mathrm{p}_{a}^{+}, \quad \mathrm{p}_{r}^{+}\right) \\
& \mathrm{uf}_{s, r}^{+}=\operatorname{sum}\left(\mathrm{uf}_{a}^{+}, \quad \mathrm{uf}_{r}^{+}\right) \\
& \mathrm{p}_{s, r}^{-}=\min \left(\mathrm{p}_{a}^{-}, \quad \mathrm{p}_{r}^{-}\right) \\
& \mathrm{uf}_{s, r}^{-}=\operatorname{sum}\left(\mathrm{uf}_{a}^{-}, \quad \mathrm{uf}_{r}^{-}\right)
\end{aligned}
$$

The settlement power is the minimum of the announcement and the offered power (cf. Eq. 9 and 11). The joint utility factor of both trading partners is calculated by adding both values (cf. Eq. 10 and 12).

Then the optimal settlement option is selected. This is done by a simple arithmetic comparison of all possible settlements regarding their utility factor. Note that the resulting utility factor sum for one or all potential settlements can be negative, if one or both of the appliances have a negative utility factor. If none of the offers can lead to increasing the overall systems utility, the Micro Grid has reached the optimized state.

\subsection{Locality-Aware Iterative Optimization}

The proposed algorithm balances out generators and loads, leading to an optimal solution. Nonetheless, local solutions are preferred since locally solved balances are not further forwarded, which depends on nodes to function as proxy nodes, that agree to virtually reduce or increase their own power consumption in favor of neighboring nodes that do not have direct communication connections to a power source.

To illustrate this property, we assume that a power generator and a power consumer are connected through a communication grid in form of a line. The algorithm takes more steps to terminate, but is still able to reach a desirable solution. A sample configuration that comprises five nodes in a line is depicted in Figure 5. Three middle nodes are simulated as battery storages, fulfilling the task of communicating the flexibility in electricity. The flexibilities of the nodes are listed in Table 1. Node 1, representing a small town, has the objective of satisfying its maximal power consumption of $2.16 \mathrm{MW}$. The three mid nodes are battery storage 1, which is fully loaded, and batteries 2 and 3, which have capabilities to charge. The fifth node represents a wind park with a current possible power output of 2.52 MW.

As depicted in Figure 6, the solution of the algorithm is for the wind mill to fully feed its power into the grid and for the town to consume its inquired electrical power. Since the wind park is providing slightly more power than required, battery 3 is set into charging state and consumes $0.36 \mathrm{MW}$.

Using the flexibility of each battery storage, the optimal solution is achieved, namely that the city is fully powered due to the high

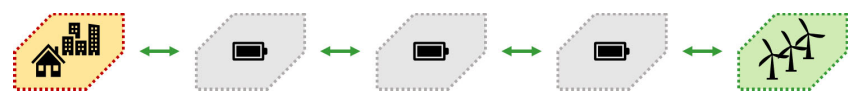

Figure 5: Example configuration in line topology

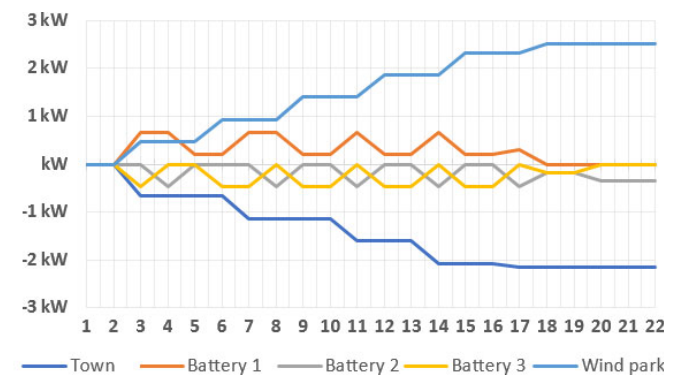

Figure 6: Result of iterative optimization.

output of the wind park. The process of reaching the solution is set out in Figure 6. In the x-axis, we show the iteration steps. The $\mathrm{y}$-axis shows the power injection/consumption in $\mathrm{kW}$. Note that in each iteration step, the overall utility only increases: The process of virtually increasing and decreasing the power consumption along the $\mathrm{x}$-axis while passing on the flexibilities is clear when looking at the behavior of the three battery nodes.

\subsection{Message Complexity}

For large grids, we recommend using clusters of the size of $\log (n)$, which would result in a cluster size of 10 for a Smart Grid with 1,000 nodes and a cluster size of 16 for a Smart Grid with 5,000 nodes.

The message complexity $O(n)$ of the proposed scheme is strongly affected by the parametrized cluster size. In each iteration, each node $n_{a}$ sends an announcement message to all other nodes in the same cluster $C_{i}$. Only those nodes $n_{r}$ respond, which might increase their utility through a pairwise settlement. Then node $n_{a}$ selects on offer $o_{r}$ and sends an according settlement message $s_{r}$ to that one node $n_{r}$. The other nodes can either be informed or timeout.

Therefore, the worst-case message complexity for one cluster with c nodes and 1 announcer is $O(c)=2 \cdot c+1=c$. Since each cluster has $c=\log (n)$ nodes and $\frac{n}{c}$ clusters are formed, the systemwide worst-case message complexity per iteration is at:

$$
O(n)=c \cdot \frac{n}{c}=n
$$

The required number of iterations $k$ varies due to multiple factors like power injection/consumption relation between the nodes and

\begin{tabular}{|c|c|c|c|c|c|}
\hline \# & Type & UF & Max Output & Result Output & Utility \\
\hline 1 & Town & 5 & $-2.16 \mathrm{MW}$ & $-2.16 \mathrm{MW}$ & 10.80 \\
\hline 2 & Battery 1 & 0 & $0.67 \mathrm{MW}$ & $0 \mathrm{MW}$ & 0 \\
\hline 3 & Battery 2 & -1 & $-0.51 \mathrm{MW}$ & $-0.36 \mathrm{MW}$ & -0.36 \\
\hline 4 & Battery 3 & -2 & $-0.46 \mathrm{MW}$ & $0 \mathrm{MW}$ & 0 \\
\hline 5 & Wind park & 9 & $2.52 \mathrm{MW}$ & $2.52 \mathrm{MW}$ & 22.68 \\
\hline \multicolumn{6}{|c|}{ Sum: } \\
\hline
\end{tabular}
topology of the cluster overlaps. In a scenario, where the clusters, generators and consumers are more-or-less evenly distributed, the

Table 1: Solution in line configuration after iterative optimization. 
number of iterations can be expected to be logarithmic to the number of clusters $O(n)=c=\log (n)$. This leads to an overall upper bound in message complexity of:

$$
O(n)=n \cdot \log (n)
$$

During the bootstrapping phase where all nodes start at 0 generation/consumption, all nodes need to calibrate. Each agent sends their initial message to $c=\log (n)$ other agents. The complexity of a "blackstart" scenario as elaborated in Section 5 has a worst-case message complexity of:

$$
O(n)=n \cdot \log ^{2}(n)
$$

\section{EXPERIMENTAL RESULTS}

The proposed algorithm is designed to work both in fully meshed communication grids as well as to be effective and resilient to varying topological conditions. This is realized by limiting the outreach of the nodes' announcements as well as the peer-to-peer character of the actual settlements. An important edge case is the line topology, which is the first investigated scenario.

In the context of Micro Grids, the desired behavior is that the power generators and loads in one area are balanced out against each others, before offering additional flexibility outside each cluster. Therefore, one main design decision of the distributed Smart Grid control algorithm is to support both clustering and the use of dedicated nodes that join multiple clusters and thereby create an overlap that allows to find an optimal solution that extends the area of influence of each DER. The following sections are focusing on simulation results for these approaches.

In addition to the results presented in Section 4.2, we set up four other simulation scenarios to evaluate the proposed distributed Smart Grid control scheme. In Section 5.1, we show the iterative behavior of the proposed algorithm in a fully-meshed communication network between 10 DERs in a low-voltage Micro Grid. Afterwards, the Micro Grid is extended to match the IEEE European Reference Low-Voltage Test Feeder Grid (IEEE LVTF) with 53 DERs distributed over the grid [5]. In Section 5.3, we assume a fully meshed communication network and evaluate the distributed Smart Grid control scheme using a linear optimization as benchmark. In the third scenario, we show that the partitioning of the Micro Grid into smaller geographical clusters actually improves the efficiency of the distributed Smart Grid control scheme due to parallelization in each cluster and also improves the locality of the optimization solution. In the last scenario in Section 5.5, we show that the algorithm can
Table 2: Subset of the DERs in the investigated Micro Grid

\begin{tabular}{r|l|r|r||r|r}
$\#$ & Type & UF & Max Output & Result Output & Utility \\
\hline \hline 1 & Aircon & 2 & $-12.00 \mathrm{~kW}$ & $0 \mathrm{~kW}$ & 0 \\
\hline 2 & Building & 5 & $-10.00 \mathrm{~kW}$ & $-10.00 \mathrm{~kW}$ & 50 \\
\hline 3 & PV & 4 & $13.00 \mathrm{~kW}$ & $13.00 \mathrm{~kW}$ & 52 \\
\hline 4 & CHP & -2 & $25.00 \mathrm{~kW}$ & $7.00 \mathrm{~kW}$ & -14 \\
\hline 5 & Building & 5 & $-12.00 \mathrm{~kW}$ & $-12.00 \mathrm{~kW}$ & 60 \\
\hline 6 & EV-Charger & 3 & $-9.00 \mathrm{~kW}$ & $-9.00 \mathrm{~kW}$ & 27 \\
\hline 7 & WPP & 4 & $27.00 \mathrm{~kW}$ & $27.00 \mathrm{~kW}$ & 108 \\
\hline 8 & CHP & -2 & $29.00 \mathrm{~kW}$ & $0 \mathrm{~kW}$ & 0 \\
\hline 9 & Crit.Infrast. & 8 & $-10.00 \mathrm{~kW}$ & $-10.00 \mathrm{~kW}$ & 80 \\
\hline 10 & Building & 2 & $-12.00 \mathrm{~kW}$ & $-6.00 \mathrm{~kW}$ & 12 \\
\hline \hline
\end{tabular}

also improve the locality of the result by adapting the clustering rather than to geographical proximity.

\subsection{Micro Grid}

The goal of the microgrid experiment is to demonstrate that the proposed distributed Smart Grid control scheme reaches an optimal solution in a fully-meshed configuration. Each DER is controlled by a dedicated agent, which runs in a virtualized environment and has their respective dedicated simulation properties. The electrical grid has a radial structure and is a sub-grid of the IEEE LVTF with ten DERs as listed in Table 2 and visualized in Figure 7.

5.1.1 Optimization Result. Figure 8 shows the iterative convergence towards the optimal solution for this subset. In each step, the sums of power consumption and injection are balanced. The

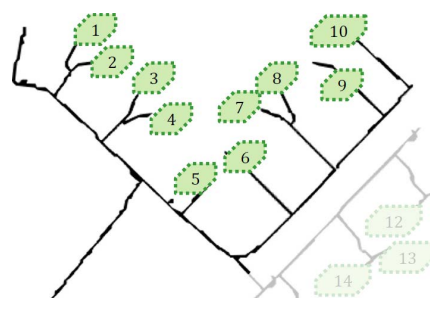

Figure 7: Part of a distribution grid with 10 DERs is simulated as an exemplary cluster.

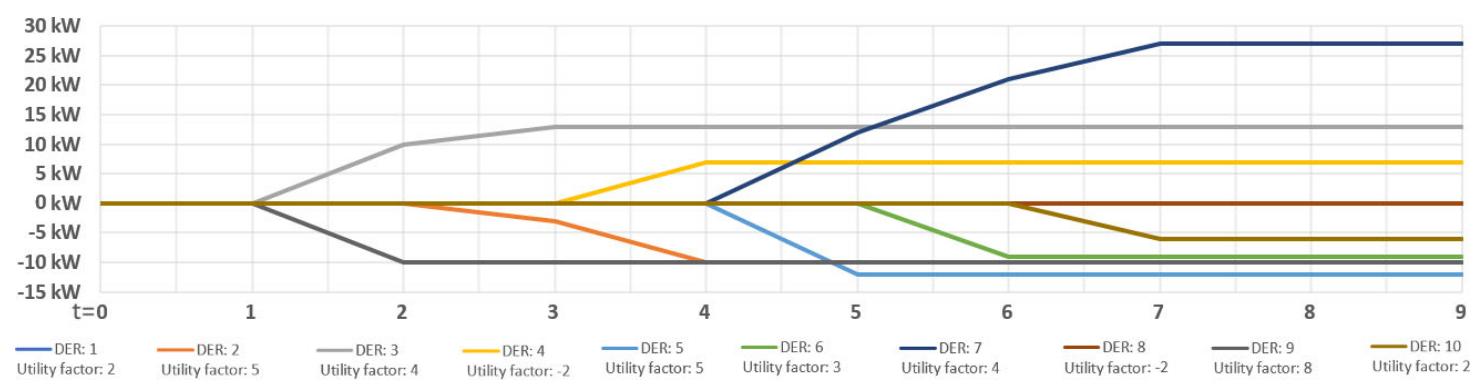

Figure 8: Grid example run with 10 nodes reaching the optimal solution. 


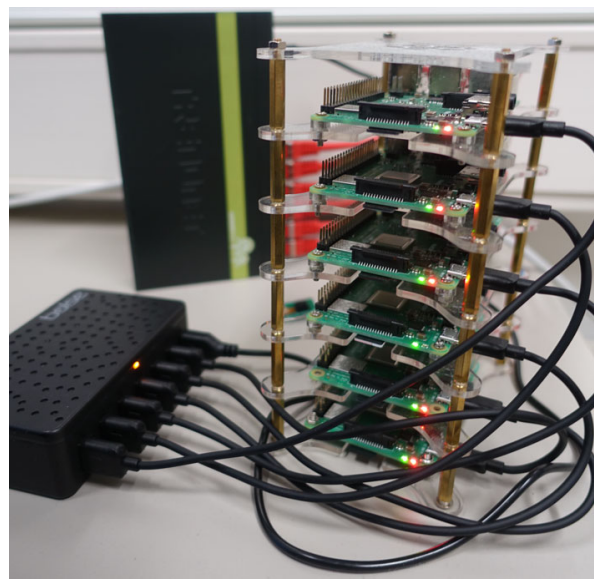

Figure 9: Hardware configuration for the control of a sub grid of ten DERs.

specific configuration results in a solution where the appliances 2, 3, 5, 6, 7 and 9 are fully operative, since their their utility factors have the highest values $(3,4,5$ and 8$)$. The DERs with lower utility factors -2 and 2 are hardly in use.

When compared with a simple linear optimization, the results are identical utility-wise, but achieved in a distributed manner without a central component.

The iterative process of the distributed Smart Grid control scheme leads to a fully balanced Micro Grid in each step. This gentle mode of operation helps to prevent sudden distortions of the voltages within the Micro Grid. Furthermore, it supports a possible isolated operation of the microgrid, where keeping the power balance would be essential.

\subsection{Controller-Hardware-in-the-Loop Setup}

In order to provide a scalable simulation, we followed the design choice to execute each agent in a separated containerized environment, which allowed only TCP/IP communication.

The proposed communication environment comprises a rack of embedded devices as pictured in Figure 9. They implement a decentralized Controller-Hardware-in-the-Loop configuration. The Raspberry Pi devices are part of the distributed simulation, connected by Ethernet cables and a switch. They are configured to each run instances of the investigated clustered peer-to-peer algorithm, using the OpenDISCO framework for distributed Smart Grid control [19].

Due to the message-based, distributed approach, the computational load on the devices remains linear to the number of hosted agents.

\subsection{Full Mesh and Linear Optimization}

For the following simulations, we increase the number of DERs to 53 and assume that they are all connected to the IEEE-LVTF grid [5]. Line data is taken from the original source, while loads are replaced by DERs according to our specific test setup: The power limits $P_{\text {lim }}$ of the DERs are chosen arbitrarily within a range of $6 \mathrm{~kW}$ to 29 $\mathrm{kW}$. In doing so, maximum power values are scaled such that the

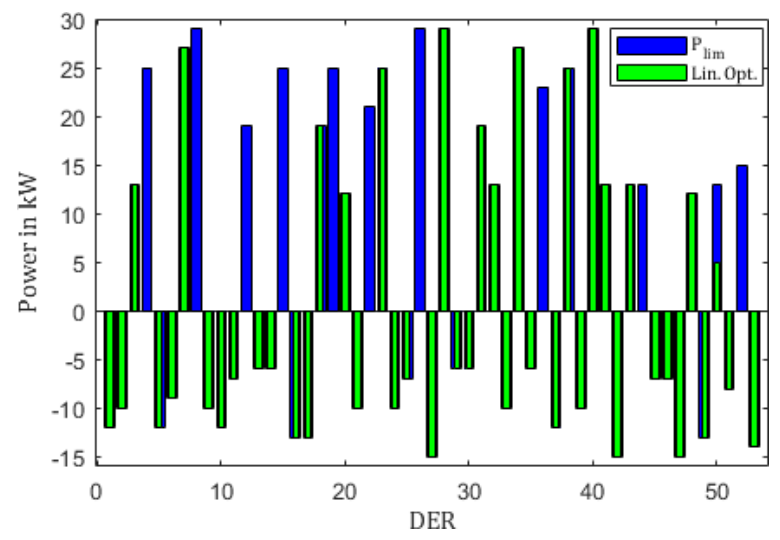

Figure 10: Result of linear optimization. The blue (darker) bars represent maximum power values $P_{\text {lim }}$, while optimization results are shown green.

total installed generation capacity equals approximately twice the installed consumer capacity. Therefore a scenario is modeled with high availability of renewable generation. It is assumed that each DER can adjust its power to any value between zero and $P_{\text {lim }}$.

We decided to evaluate the distributed Smart Grid control scheme against the IEEE LVTF as illustrated in Figure 1, and individually simulate DERs with dedicated properties at each of the 53 relevant nodes.

The behavior of the grid agents is simulated using a single server, which operates the agents in docker containers. As a benchmark to evaluate the performance of distributed Smart Grid control scheme, the DER's optimal power values for maximization of the overall utility are determined by a linear optimization using MATLAB's linprog-function. The results are plotted in Figure 10. While all loads are fully supplied, only generators with high utility factor are activated. The corresponding overall utility value is 2,372 . The resulting solution of the autonomous DERs again has the same global utility value as the linear optimization scheme.

\subsection{Geographical Clustering}

The fully meshed communication structure utilized in the previous simulations (cf. Section 5.3) is disadvantageous in terms of scalability, as the number of peer-to-peer connections increases exponentially with the number of DERs in the grid. This implies that in case of new DERs being installed in the grid, communication has to be established to all existing devices. We investigate a distributed solution, with the original 53 DERs being grouped into 8 clusters as shown in Figure 11. The clusters are implemented by software-defined networking (SDN). In our experiments, we utilize the SDN implementation provided by the docker project. Within each cluster, a full communication structure is assumed, and the distributed Smart Grid control scheme is employed to match producers and consumers. Communication across cluster boundaries is only established by certain border nodes (translator nodes) that belong to two neighboring clusters at the same time. If a flexibility offer does not find a corresponding bid within one cluster, the translator node issues a respective offer in the neighboring cluster. 


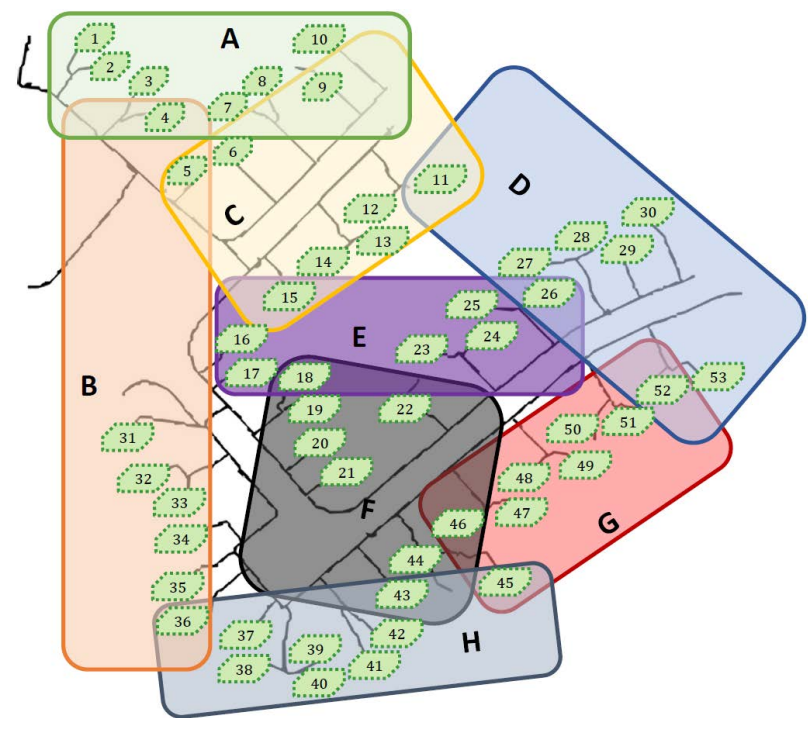

Figure 11: Distribution grid with 8 geographically picked clusters.

In terms of overall utility, the clustered communication scheme results in a slightly lower value of 2.164 , which equals $84.6 \%$ of the maximum achievable utility according to Section 5.3. This decrease stems from the fact that the algorithm now preferably matches consumers and generators within the same cluster. At most, the offers are matched across one border by the overlapping nodes' agents. Therefore, potentially more utility-increasing offers from farther clusters are neglected. On the other hand, the clustered scheme decreases convergence time due to parallel match-making, reduces communication requirements and increases scalability, as new agents could easily be integrated. Furthermore, the local approach has the potential to increase grid compatibility, as detailed below.

Table 3: Load flow calculation results for global and clustered communication schemes.

\begin{tabular}{l|r|r} 
Quantity & global & clustered \\
\hline \hline Total grid losses & $4.07 \mathrm{~kW}$ & $2.55 \mathrm{~kW}$ \\
\hline Relative grid losses & $1.45 \%$ & $1.03 \%$ \\
\hline Total power consumption & $281 \mathrm{~kW}$ & $247 \mathrm{~kW}$ \\
\hline Utility sum & $2,557.0$ & $2,164.0$ \\
\hline Min. voltage magnitude & $99.1 \%$ & $99.6 \%$ \\
\hline Max. voltage magnitude & $103.4 \%$ & $102.4 \%$ \\
\hline Max. line current magnitude & $276.0 \mathrm{~A}$ & $162.6 \mathrm{~A}$ \\
\hline Current magnitude - line 21 & $89.5 \mathrm{~A}$ & $58.2 \mathrm{~A}$ \\
\hline Current magnitude - line 22 & $79.0 \mathrm{~A}$ & $0.5 \mathrm{~A}$ \\
\hline Current magnitude - line 23 & $168.5 \mathrm{~A}$ & $58.7 \mathrm{~A}$ \\
\hline Current magnitude - line 51 & $276.0 \mathrm{~A}$ & $162.6 \mathrm{~A}$ \\
\hline Current magnitude - line 52 & $124.8 \mathrm{~A}$ & $137.8 \mathrm{~A}$ \\
\hline Current magnitude - line 53 & $151.1 \mathrm{~A}$ & $24.7 \mathrm{~A}$ \\
\hline & \multicolumn{2}{|c}{}
\end{tabular}

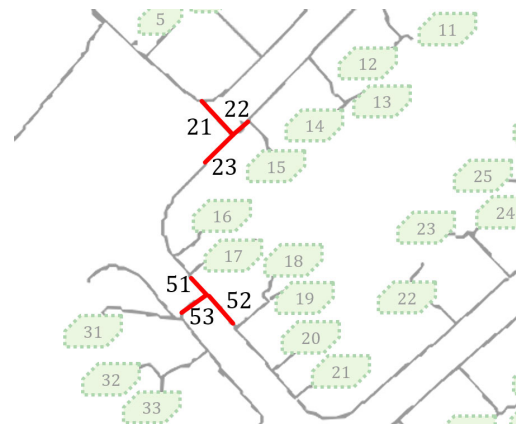

Figure 12: Critical distribution lines (cf. Table 3 and 4).

5.4.1 Grid Capacity. So far, we have ignored the actual power grid capacity, which is expressed in terms of power grid constraints as explained in Section 2. To evaluate the impact of the distributed Smart Grid control scheme results for the different communication architectures (fully meshed vs. distributed) on the power grid utilization, we compare load flow calculations for the power profiles resulting from both cases. The load flow calculations are performed in MATLAB under the assumption that all loads are symmetric, which allows to consider one of the three phases only. Therefore, all results refer to one single phase. In addition, it is assumed that the grid is connected to the overlying medium voltage grid via the substation transformer. The transformer's secondary voltage equals its nominal value of $416 \mathrm{~V}$. All DERs are assumed to operate with unity power factor, i.e. they neither consume nor provide reactive power. Relevant results are summarized in Table 3.

The upper three rows indicate that the distributed algorithm is advantageous in terms of grid losses, which are the sum of the electrical distribution lines' active power consumption. Note that as the distributed Smart Grid control scheme keeps electric power consumption and injection balanced out, grid losses are supplied by the substation transformer. The relative losses, which equal the total losses divided by the consumers' total power consumption, are decreased by almost $30 \%$ with respect to the fully-meshed topology without clustering. This corresponds to the mean value of line current magnitude, which is reduced in a similar proportion. Furthermore, the voltage profile within the grid is flattened: the maximum voltage is decreased from $103.4 \%$ to $102.4 \%$ of its nominal value, while the minimum voltage is increased from $99.1 \%$ to $99.6 \%$. In any case, maximum voltage deviations are well the usual limit of $+/-10 \%$ of the nominal voltage, as e.g. defined in the IEC 60038 standard [6].

\subsection{Electric Grid Inspired Clustering}

While other balancing effects may play a role in the exemplary setup investigated here, the general tendency of grid loss reduction presumably stems from the increased locality: in the distributed case, flexibility matches are preferably made within clusters. Therefore, power flows between clusters that might be geographically distant are naturally avoided by the distributed algorithm. The line current magnitude comparison for selected distribution lines provided in the lower six rows of Table 3 , using the line numbering according to Figure 12, supports this assumption, as the current 


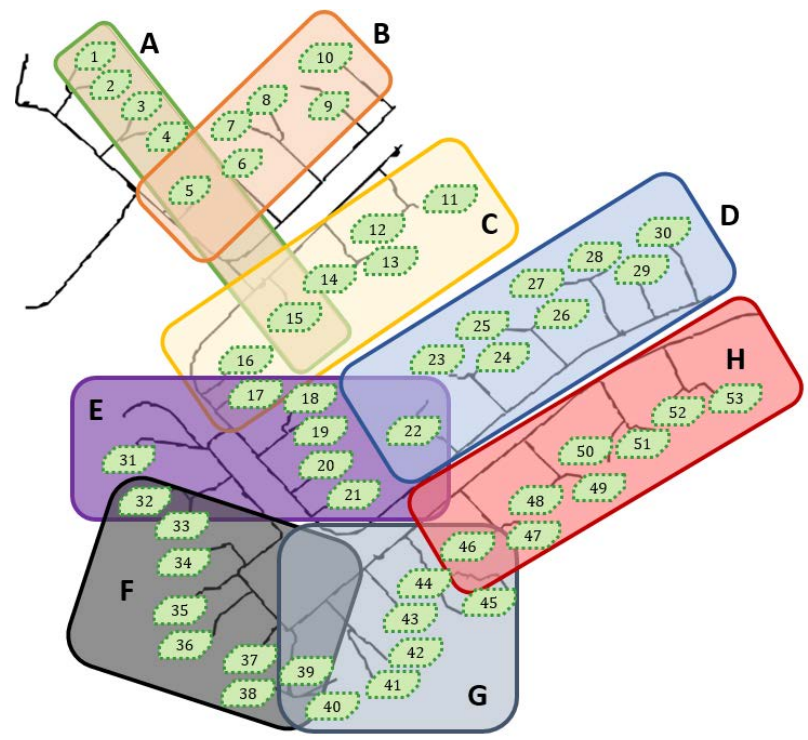

Figure 13: Distribution grid with clusters designed according the distribution lines

magnitude is significantly lower on most of these central junction lines in the distributed case than in the central case. It is noticeable that this is observed even though short distribution line lengths within clusters were not a criterion in the cluster design, as can be seen in Figure 11. For this reason, in the next subsection we investigate whether further improvements regarding distribution line utilization and grid loss reduction can be achieved by clustering the grid nodes according to the distribution lines.

We apply the distributed Smart Grid control scheme to the IEEE LVTF with 53 DERs again, using the same maximum power values and utility factors as in the previous simulations. For this experiment we introduce a new clustering, where the clusters are formed under constraints of the power grid topology, aiming at short distribution lines among nodes within each cluster. This configuration is shown in Figure 13 and will be referred to as clustering B.

Compared to the clustering A from Figure 11, it should be noted that fewer overlaps exist in clustering B: three clusters (namely B, $\mathrm{D}$ and $\mathrm{H}$ ) only contain one interface node to another cluster.

Again, a load flow calculation is made with the resulting power injection and consumption values to evaluate the impact on the electric grid. Table 4 shows a comparison of the relevant quantities for the geographical clustering from the previous section and the grid-oriented clustering. Though the overall system utility drops by $5.5 \%$ in respect to the linear optimization solution, it can be noted that grid losses are further reduced by approx. $10 \%$. While voltage magnitude deviations from nominal value remain small, the maximum line current magnitude is reduced by $31 \%$. Line current magnitudes on the central junction line shown in Figure 12 are significantly smoothed out. These results indicate that the distributed Smart Grid control scheme with grid-oriented clustering indeed leads to a more grid-serving power profile, without requiring to explicitly consider electricity flow constraints.

\section{DISCUSSION}

The proposed algorithm works in an easy-to-understand yet effective way for electricity distribution grids and aims to contribute to a concrete transition path between the current electric grid control and future resilient Smart Grids.

\subsection{Requirements Evaluation}

The requirements postulated in Section 2 are met as follows:

Locality-Awareness: The presented optimization scheme is inherently locality-aware due to the restriction of communication which requires an iterative process to reach non-local solutions. The main indicator for locality-awareness used in the experiments are the relative grid losses, which could be reduced from $1.45 \%$ to $0.94 \%$ which itself is a reduction by $35.17 \%$ and shows the locality-awareness.

Tie-Breakingness: Although multiple equivalently optimal solutions may exist regarding the optimization of overall utility, the presented scheme selects the most-local solution. In the case of more than one possibility within one cluster, the algorithm does not stall but instead is capable of selecting one of the equally suited choices.

Scalability: The agent-based approach to execute each process in a separated environment results in low inter-dependencies between the components. Due to the SoS approach and the concept of breaking up the complete Smart Grid optimization task into clusters, the computational requirements for each agent remain constant regardless of the number of participating nodes.

Iterativeness: We show that the optimization scheme only selects choices that increase the overall utility, so that each iteration step works towards an optimal solution. At the same time the presented scheme assures a balanced result at each intermediate step. In case of aborting the distributed process, a (sub-optimal) solution (iteration) is reached in any case.

Measurability: The algorithm uses two indicators for its efficiency, which are the overall utility and grid losses.

Table 4: Load flow calculation results for geographical and grid-oriented clustering schemes.

\begin{tabular}{l|r|r} 
Quantity & geographical & grid-oriented \\
\hline \hline Total grid losses & $2.55 \mathrm{~kW}$ & $2.09 \mathrm{~kW}$ \\
\hline Relative grid losses & $1.03 \%$ & $0.94 \%$ \\
\hline Total power consumption & $247 \mathrm{~kW}$ & $223 \mathrm{~kW}$ \\
\hline Utility sum & $2,164.0$ & $2,023.0$ \\
\hline Min. voltage magnitude & $99.6 \%$ & $98.7 \%$ \\
\hline Max. voltage magnitude & $102.4 \%$ & $101.1 \%$ \\
\hline Max. line current magnitude & $162.6 \mathrm{~A}$ & $111.2 \mathrm{~A}$ \\
\hline Current magnitude - line 21 & $58.2 \mathrm{~A}$ & $6.87 \mathrm{~A}$ \\
\hline Current magnitude - line 22 & $0.5 \mathrm{~A}$ & $53.49 \mathrm{~A}$ \\
\hline Current magnitude - line 23 & $58.7 \mathrm{~A}$ & $60.33 \mathrm{~A}$ \\
\hline Current magnitude - line 51 & $162.6 \mathrm{~A}$ & $48.54 \mathrm{~A}$ \\
\hline Current magnitude - line 52 & $137.8 \mathrm{~A}$ & $52.3 \mathrm{~A}$ \\
\hline Current magnitude - line 53 & $24.7 \mathrm{~A}$ & $3.78 \mathrm{~A}$ \\
\hline
\end{tabular}




\subsection{Conclusion}

We present a distributed Smart Grid control algorithm that achieves pairwise balance of power consumption and injection. The behavior and power flow constraints of the proposed scheme are evaluated and improved using two different clustering approaches. The proposed algorithm is capable of finding a compromise between a utility-wise optimal solution and maintaining locality-awareness. The electrical efficiency is increased by a reduction of relative losses, which equal the total losses divided by the consumers' total power consumption, by almost $30 \%$.

\subsection{Future Work}

For future research, we want to use the algorithm to optimize multiple grids, connected by medium voltage lines. Therefore we plan to extend the algorithm to support hierarchical functions. We plan to propose a concept with a better differentiation between the nodes capabilities, computational power, communication link quality and their respective responsibilities in the grid, e.g. by introducing dedicated aggregator nodes, that can take more centralized roles.

The role of locality has been investigated using load-flow computations. In future research, we want to elaborate a measure for locality which can be evaluated in a distributed manner, so that the algorithm can improve itself according to its locality in terms of adjustment to the electrical grid. Further, we are planning to propose fine-grained mechanisms to configure the algorithm such that the compromise between locality and utility-optimization can be easily adjusted according to applicable real-world requirements. This includes an extended review of clustering schemes and cluster sizes for this algorithm.

\section{ACKNOWLEDGMENTS}

This work was funded by the Federal Ministry of Economics and Energy of Germany (BMWi).

\section{REFERENCES}

[1] E. Dall'Anese and A. Simonetto. 2018. Optimal Power Flow Pursuit. IEEE Transactions on Smart Grid 9, 2 (March 2018), 942-952. https://doi.org/10.1109/TSG. 2016.2571982

[2] K. Eger and U. Killat. 2007. Resource pricing in peer-to-peer networks. IEEE Communications Letters 11, 1 (Jan 2007), 82-84. https://doi.org/10.1109/LCOMM 2007.061094

[3] Irene Eusgeld, Cen Nan, and Sven Dietz. 2011. âĂIJSystem-of-systemsâĂİ ap proach for interdependent critical infrastructures. Reliability Engineering \& System Safety 96, 6 (2011), 679-686.

[4] M. N. Faqiry and S. Das. 2019. Distributed Bilevel Energy Allocation Mechanism With Grid Constraints and Hidden User Information. IEEE Transactions on Smart Grid 10, 2 (March 2019), 1869-1879. https://doi.org/10.1109/TSG.2017.2779826
[5] IEEE PES Distribution Systems Analysis Subcommittee Radial Test Feeders. 2015. European Low Voltage Test Feeder. http://sites.ieee.org/pes-testfeeders/ resources/. Accessed: 2018-10-10.

[6] German Commission for Electrical, Electronic and Information Technologies of DIN and VDE. 2012-04. DIN EN 60038, VDE 0175-1: CENELEC standard voltages (IEC 60038:2009, modified); German version EN 60038:2011.

[7] John Hetzer, C Yu David, and Kalu Bhattarai. 2008. An economic dispatch model incorporating wind power. IEEE Transactions on energy conversion 23, 2 (2008), 603-611.

[8] S. H. Low. 2014. Convex Relaxation of Optimal Power Flow-Part I: Formulations and Equivalence. IEEE Transactions on Control of Network Systems 1, 1 (March 2014), 15-27. https://doi.org/10.1109/TCNS.2014.2309732

[9] Magdi S Mahmoud, Mohamed Saif Ur Rahman, and MAL-Sunni Fouad. 2015. Review of microgrid architectures-a system of systems perspective. IET Renewable Power Generation 9, 8 (2015), 1064-1078.

[10] Mark W Maier. 1998. Architecting principles for systems-of-systems. Systems Engineering: The fournal of the International Council on Systems Engineering 1, 4 (1998), 267-284.

[11] Saurabh Mittal, Mark Ruth, Annabelle Pratt, Monte Lunacek, Dheepak Krishnamurthy, and Wesley Jones. 2015. A system-of-systems approach for integrated energy systems modeling and simulation. In Proceedings of the Conference on Summer Computer Simulation. Society for Computer Simulation International, $1-10$.

[12] Daniel K Molzahn, Florian Dörfler, Henrik Sandberg, Steven H Low, Sambuddha Chakrabarti, Ross Baldick, and Javad Lavaei. 2017. A survey of distributed optimization and control algorithms for electric power systems. IEEE Transactions on Smart Grid 8, 6 (2017), 2941-2962.

[13] Clay G Nesler, Michael G Andrew, John I Ruiz, and David B Busch. 2010. Electrical demand response using energy storage in vehicles and buildings. US Patent App. 12/324,687.

[14] Jennifer Pérez, Jessica Díaz, Juan Garbajosa, Agustín Yagüe, Eloy Gonzalez, and Mercedes Lopez-Perea. 2013. Large-scale smart grids as system of systems. In Proceedings of the First International Workshop on Software Engineering for Systems-of-Systems. ACM, 38-42.

[15] Hajir Pourbabak, Tao Chen, and Wencong Su. 2016. Consensus-based distributed control for economic operation of distribution grid with multiple consumers and prosumers. In 2016 IEEE Power and Energy Society General Meeting (PESGM).

[16] Navid Rahbari-Asr, Unnati Ojha, Ziang Zhang, and Mo-Yuen Chow. 2014. Incremental welfare consensus algorithm for cooperative distributed generation/demand response in smart grid. IEEE Transactions on Smart Grid 5, 6 (2014).

[17] Saifur Rahman and Yonael Teklu. 2000. Role of the electric vehicle as a distributed resource. In 2000 IEEE Power Engineering Society Winter Meeting. Conference Proceedings (Cat. No. 00CH37077), Vol. 1. IEEE, 528-533.

[18] Lingyu Ren, Yanyuan Qin, Yan Li, Peng Zhang, Bing Wang, Peter B Luh, Song Han, Taofeek Orekan, and Tao Gong. 2018. Enabling resilient distributed power sharing in networked microgrids through software defined networking. Applied Energy 210 (2018), 1251-1265.

[19] Marius Stübs and Kevin Köster. 2018. OpenDISCO-Open simulation framework for distributed smart grid control. Energy Informatics 1, 1 (2018), 343-348.

[20] Mathias Uslar, Sebastian Rohjans, Christian Neureiter, Filip Pröstl Andrén, Jorge Velasquez, Cornelius Steinbrink, Venizelos Efthymiou, Gianluigi Migliavacca, Seppo Horsmanheimo, Helfried Brunner, et al. 2019. Applying the smart grid architecture model for designing and validating system-of-systems in the power and energy domain: A European perspective. Energies 12, 2 (2019), 258.

[21] Hongye Wang, Carlos E Murillo-Sanchez, Ray D Zimmerman, and Robert J Thomas. 2007. On computational issues of market-based optimal power flow. IEEE Transactions on Power Systems 22, 3 (2007), 1185-1193.

[22] Kun Wang, Xiaoxuan Hu, Huining Li, Peng Li, Deze Zeng, and Song Guo. 2017. A survey on energy internet communications for sustainability. IEEE Transactions on Sustainable Computing 2, 3 (2017), 231-254.

[23] Chengcheng Zhao, Jianping He, Peng Cheng, and Jiming Chen. 2017. Consensusbased energy management in smart grid with transmission losses and directed communication. IEEE Transactions on smart grid 8, 5 (2017), 2049-2061. 